\title{
COVID-19 in Refractory Myasthenia Gravis- A Case Report of Successful Outcome
}

\author{
Swathi Beladakere Ramaswamy ${ }^{\mathrm{a}, *}$ and Raghav Govindarajan ${ }^{\mathrm{b}}$ \\ ${ }^{a}$ Department of Neurology, University of Missouri, Columbia, Missouri \\ ${ }^{\mathrm{b}}$ Department of Neurology, University of Missouri, Columbia, Missouri
}

\begin{abstract}
This is a brief report of a patient who has refractory Myasthenia Gravis, on multiple long-term immunosuppressive therapies and contracted COVID-19 during this 2020 pandemic. She was quarantined for total of 14 days and recovered successfully without any complications (no myasthenia exacerbation or crisis, no COVID-19 related complications), with no changes to her immunosuppressive therapy. Treatment of MG patients with COVID-19 needs to be tailored to individual patient.
\end{abstract}

Keywords: Refractory Myasthenia Gravis, Plasmapheresis, immunosuppression, COVID-19, outcome

\section{INTRODUCTION}

Myasthenia gravis is an autoimmune disease affecting the neuromuscular junction and causing fatigable ocular, limb, and bulbar weakness. Patients can be in remission with treatment and relapse to exacerbation with worsening of symptoms and in $15 \%$ of cases, patient experience a neurologic emergency characterized by restrictive respiratory failure known as myasthenic crisis. This exacerbation/crisis is mostly precipitated by concurrent infection [1].

In the recent global pandemic from novel coronavirus (COVID-19/SARS-CoV2), myasthenic patients can be considered high risk due to chronic immunosuppression. This case report describes myasthenic patient who was infected COVID-19 and

\footnotetext{
*Correspondence to: Dr Swathi Beladakere Ramaswamy, Department of Neurology, University of Missouri, CE515, CS \& E building, Columbia, Missouri 65212, 573882 2260. E-mail: ramaswamys@health.missouri.edu.
}

recovered without myasthenic crisis/exacerbation, and no COVID-19 complications despite chronic immunomodulatory therapy.

\section{CASE}

A 42-year-old Caucasian female was diagnosed with acetylcholine receptor antibody positive oculobulbar myasthenia gravis nine months ago. Her initial presenting symptoms were drooping eyelids, double vision and difficulty swallowing with diurnal variation and characteristic fluctuation of symptoms. She initially failed her bedside swallow evaluation with notable aspiration to thin liquids on modified barium swallow (MBS). She underwent plasmapheresis with complete resolution of symptoms following IVIG as her swallowing functions didn't improve with the latter. Eventually patient's repetitive nerve stimulation study and antibody titers confirmed the diagnosis of postsynaptic neuromuscular junction disorder. 
Prior to discharge patient was started on pyridostigmine $60 \mathrm{mg} 4$ times daily, gradually up-titrated dose of prednisone $30 \mathrm{mg}$ daily and mycophenolate 1000 milligrams twice daily. Her past medical history included generalized anxiety disorder and allergic rhinitis for which she continued to follow with her primary physician at discharge.

During her routine neurology clinic follow-up patient was noted to have recurrence of drooping eyelids and change in voice with concerns of impending myasthenia exacerbation. As she responded very well to plasmapheresis compared to IVIG, she was started on regular plasma exchange, 3 exchanges every 4 weeks. She continued to take rest of her medications including prednisone $30 \mathrm{mg}$ daily and mycophenolate 1000 milligrams twice daily. Patient's CT chest showed $6.7 \mathrm{~cm}$ lobulated soft tissue mass in anterior mediastinum with no local invasion, consistent with thymoma or thymic carcinoma. She was referred to cardiothoracic surgery for possible thymectomy (which had been put on hold due to patient request).

Four weeks ago, patient presented to emergency department (ED) with fever, chills, cough with minimal clear sputum production, exertional shortness of breath, decreased sense of taste and smell, decreased appetite going on for the past 5 days. She had traveled to nearby city two weeks prior to her presentation. There was no clear history of exposure to sick and no history of similar symptoms in friends or family she came in contact with. Patient's chest x-ray showed patchy infiltrates in left lower lobe concerning for infection. Patient's labs showed elevated white count $\left(12.32 \times 10^{9} / \mathrm{L}\right)$ with lymphopenia $\left(0.78 \times 10^{9} / \mathrm{L}\right)$, respiratory pathogen panel including influenza $\mathrm{A} / \mathrm{B}$, Streptococcus pneumonia, urine Legionella came back negative. She was tested for COVID-19 RTPCR with CDC primers, which came back positive. A bedside negative inspiratory force (NIF) obtained was $65 \mathrm{~cm} \mathrm{H}_{2} \mathrm{O}$. Patient was discharged from emergency department with instructions to self-quarantine for next fourteen days, follow CDC (Center for Disease Control and Prevention) guidelines of hand hygiene and contact precautions to prevent spread of infection and clear instructions to return to emergency department if her myasthenia symptoms or respiratory symptoms worsened. Patient's immunomodulatory therapy including steroids and mycophenolate were continued during this time (patient had been on this therapy for seven months). Her plasmapheresis was deferred to post quarantine period in view of preventing spread of infection to others.
Patient recovered from COVID-19 infection with no complications. She did not develop any symptoms of myasthenic crisis or myasthenia exacerbation during her course of infection. No changes to her immunosuppressive medications were made during the course of infection.

\section{DISCUSSION}

Myasthenia gravis (MG) is a prototype autoimmune disease where the muscle weakness is induced by autoantibodies binding to the postsynaptic region and impairing the function of acetylcholine receptors (AChR) $[1,2]$. MG is treatable with immunomodulation from long-term immunosuppressive drugs, IV immunoglobulin (IVIg), and plasmapheresis [1-3] In approximately $15 \%$ of patients, severe weakness of respiratory muscles causes restrictive respiratory failure (ie, myasthenic crisis), a true neurologic emergency that requires prompt treatment in an intensive care setting [1-8].

Among various trigger factors for MG exacerbation and crisis infections are the most common cause [5-9]. Respiratory infections may occur when respiratory muscle weakness causes atelectasis due to inadequate ventilation, stagnation of secretions and dysphagia causing recurrent aspirations [3-5, 10]. Long term immunosuppressive drug therapy effectively reduces immune reactivity broadly, including the reactivity against microbial antigens $[4,5]$. Plasmapheresis and intravenous immunoglobulin are the preferred treatments for an MG crisis [5-8].

In the recent global pandemic from novel coronavirus (COVID-19/SARS-CoV2), myasthenic patients can be considered high risk in case of chronic immunosuppression and in case of respiratory muscle weakness [11-14]. To aid management of MG during this global pandemic a panel of experts published a consensus statement. According to the MG expert panel the decision whether to continue patient's immunomodulatory therapy should be individualized and made collaboratively between patient and primary neurologist [16].

There is no scientific evidence to suggest that symptomatic therapies like pyridostigmine increases the risk of infection and should not be discontinued unless there are other clinical reasons to do so [16]. Altering or stopping immunomodulatory therapy abruptly carries potentially severe increase in disease activity, relapse, and exacerbation/crisis and hence should be based on specific risks (e.g., age, 
Table 1

MG expert panel's consensus on immunosuppressive therapy during COVID 19 infection ${ }^{16}$

\begin{tabular}{|c|c|c|}
\hline Immunosuppressive drug & Mechanism of action & $\begin{array}{l}\text { MG expert panels consensus whether to continue or } \\
\text { discontinue during COVID } 19\end{array}$ \\
\hline Azathioprine & $\begin{array}{l}\text { Purine analog that inhibits the synthesis of nucleic } \\
\text { acids and interferes with } \mathrm{B} / \mathrm{T} \text { cell proliferation. }\end{array}$ & $\begin{array}{l}\text { Rarely discontinued. Decision based on age, } \\
\text { comorbid health conditions, location. }\end{array}$ \\
\hline Mycophenolate & $\begin{array}{l}\text { Blocks purine synthesis and inhibits lymphocytic } \\
\text { proliferation. }\end{array}$ & $\begin{array}{l}\text { Rarely discontinued. Decision based on age, } \\
\text { comorbid health conditions, location. }\end{array}$ \\
\hline Cyclosporine & $\begin{array}{l}\text { Inhibits calcineurin, impairs the production of } \\
\text { interleukin-2, inhibits the function of T helper } \\
\text { cells, and dampens T lymphocyte-dependent } \\
\text { immune responses. }\end{array}$ & $\begin{array}{l}\text { Usually held until the patient recovers from } \\
\text { infection. }\end{array}$ \\
\hline Methotrexate & $\begin{array}{l}\text { Folate antimetabolite that inhibits DNA synthesis, } \\
\text { repair, and cellular replication. }\end{array}$ & $\begin{array}{l}\text { Usually held until the patient recovers from } \\
\text { infection. }\end{array}$ \\
\hline Rituximab & $\begin{array}{l}\text { Immunoglobulin G1 (IgG1) monoclonal antibody } \\
\text { (mAb) against CD20 surface receptors and acts by } \\
\text { depleting CD20-positive B cells. }\end{array}$ & $\begin{array}{l}\text { Usually discontinued during an active infection and } \\
\text { avoid initiating medication during active infection. }\end{array}$ \\
\hline
\end{tabular}

comorbid health conditions, location) and benefits. $[15,16]$.

Based on regional data available it is better to switch infusion therapies to home if there is greater risk of contracting infection in hospitals and infusion centers [16]. The Plasma Protein Therapeutics Association (PPTA) has issued a statement stating that a lipid envelope virus (SARS-CoV-2), is not a concern for the safety of plasma protein therapies as strict screening procedures for plasma donors and the established processes of virus inactivation and removal during the manufacturing of plasmaderived products is maintained [17]. Hence, IVIG and plasmapheresis can be used during MG exacerbation and crisis without any hesitation [16, 17].

Most patients who develop COVID-19 have mild disease and it is recommended to continue the current best practice standard of care for MG [16]. There might be a need to increase the dose of corticosteroids as in standard infection/stress steroid protocols [16]. However, if the symptoms are severe (requiring hospitalization) it may be necessary to consider pausing current immunosuppression temporarily, especially if there is concurrent infections/sepsis [16]. MG expert panel came to a consensus that it may be better to hold off on B-cell depleting agents (rituximab) under such conditions and individualize the continuation of other standard immunosuppressive agents (azathioprine, mycophenolate) [16]. There is currently no evidence to support that inhibition of complement through eculizumab increases susceptibility to COVID infection or its outcome [16]. Table 1 briefly outlines the mechanism of action of various immunosuppressive therapies used in MG and the MG expert panel's consensus regarding whether to continue or discontinue their use during global pandemic.
Patient mentioned in our case report was on monthly plasmapheresis, daily prednisone and mycophenolate at the time she contracted COVID19 infection. Her MG was relatively controlled with treatment and at baseline she didn't have any respiratory dysfunction. Though at presentation, patient had exertional shortness of breath and patchy infiltration on chest X ray, her initial NIF was well above normal level and there were no concerns for impending respiratory failure. Hence, she was discharged from emergency department to self-quarantine at home. As described above patient's initial MG presentation was oculo-bulbar type and required aggressive treatment in the beginning to achieve remission. The risk of exacerbation/crisis was high in her with stoppage of therapy, hence, the decision to continue her immunomodulatory therapy was made. She tolerated the therapy well and no complications were noted. Her plasmaphereses were deferred to post quarantine period only to prevent her from spreading the infection to others.

\section{CONCLUSION}

Treatment of MG patients with COVID-19 needs to be tailored to individual patient. Most decisions for treatment escalation have to be individualized based on the relative severity of COVID-19 and MG. Continuation of immunomodulatory therapies should be based on collaborative decision-making model involving patient and primary neurologist.

\section{CONFLICT OF INTEREST}

The authors have no conflict of interest to report. 


\section{REFERENCES}

[1] Ciafaloni E. Myasthenia Gravis and Congenital Myasthenic syndromes. Continuum (Minneap Minn). 2019;25(6):176784.

[2] Gilhus NE, Verschuuren JJ. Myasthenia gravis: subgroup classification and therapeutic strategies. Lancet Neurol. 2015;14:1023-36.

[3] Gilhus NE. Myasthenia gravis. N Engl J Med 2016;375:2570-2581.

[4] Gilhus NE, Nacu A, Andersen JB, Owe JF. Myasthenia gravis and risks for comorbidity. Eur J Neurol. 2015;22:1723.

[5] Wendell LC, Levine JM. Myasthenic crisis. Neurohospitalist. 2011;1(1):16-22.

[6] Jani-Acsadi A, Lisak RP. Myasthenic crisis: guidelines for prevention and treatment. J Neurol Sci. 2007;261:127-133.

[7] Chaudhuri A, Behan P. Myasthenic crisis. QJM. 2008; 102:97-107.

[8] Thomas C, Mayer S, Gungor Y, et al. Myasthenic crisis clinical features, mortality, complications, and risk factors for prolonged intubation. Neurology. 1997;48:1253-60.

[9] Gummi RR, Kukulka NA, Deroche CB, Govindarajan R. Factors associated with acute exacerbation of Myasthenia Gravis. Muscle Nerve. 2019;60(6):693-9.

[10] Gilhus NE, Romi F, Hong Y, Skeie GO. Myasthenia gravis and infectious disease. Journal of Neurology. 2018;265:1251-58.
[11] Cascella M, Rajnik M, Cuomo A, et al. Features, Evaluation and Treatment Coronavirus (COVID-19) [Updated 2020 Mar 20].

[12] Coronavirus Disease 2019 (COVID-19). Centers for Disease Control and Prevention. https://www.cdc.gov/ coronavirus/2019-ncov/index.html. Accessed April 10, 2020.

[13] Coronavirus. World Health Organization. https://www.who. int/emergencies/diseases/novel-coronavirus-2019/events as they happened; Accessed April 10, 2020.

[14] Channappanavar R, Zhao J, Perlman S. T-cell-mediated immune response to respiratory coronaviruses. Immunol Res. 2014;59(1-3):118-28.

[15] Cao M, Katz S, Simpson Greene E, Davis E, Verma A, Day JW, Wolfe L. Pulmonary Support for Neuromuscular Disease Patients During COVID19 Pandemic.

[16] International MG/COVID-19 Working Group, Jacob S, Muppidi S et al. Guidance for the management of myasthenia gravis (MG) and Lambert-Eaton myasthenic syndrome (LEMS) during the COVID-19 pandemic. J Neurol Sci. 2020;25:116803.

[17] Menger A. Plasma Protein Therapeutics Association. Home - Plasma Protein Therapeutics Association (PPTA). https://www.pptaglobal.org/. Published September 24, 2019. Accessed April 10, 2020. 